\title{
Design of a Flywheel Control for Fuel Cell Vehicles
}

\author{
Chung-Neng Huang* and Yui-Sung Chen \\ National University of Tainan, 33 Sec. 2, Shu-Lin St., Tainan 70005, Taiwan
}

(Received December 22, 2015; accepted May 16, 2016)

Keywords: magnetic flywheel, fuel cell, hybrid powertrain, fuzzy control, system stability

This paper proposes a novel powertrain with a magnetic flywheel to increase the fuel-cell output for electric vehicles (EVs). This design enables a constant fuel cell output to prevent polarization loss occurring in rapid acceleration and deceleration driving. The simulation shows that this design can effectively support the fuel cell to meet the EV power demands and improve the fuel cell efficiency to provide for better performance.

\section{Introduction}

The structure of a fuel cell is less complicated than that of a conventional gas or diesel engine and also has less corrosion and fewer structural weaknesses. Thus, fuel cells are one of the most promising alternative power sources for electric vehicles (EVs). However, there are some drawbacks that need to be addressed, including slow start-up and power response, and no bidirectional energy flow to enable regeneration by using braking energy. To overcome these drawbacks, the integration of fuel cells with energy storage devices such as batteries or supercapacitors is necessary, and representative examples of this approach include the Toyota fuel cell hybrid vehicles (FCHVs). ${ }^{(1)}$ Although these solutions can mitigate the drawbacks of fuel cells, they also bring new problems, such as high self-discharge, low energy density, and voltage.

Instead of supercapacitors and traditional batteries, by applying high-strength and light-weight composite materials and new technologies of power electronics, lithium-ion batteries, bearings, and controls, the magnetic flywheel system (MFS) is proposed as an alternative for energy storage. The MFS has many advantages, including higher energy storage density, lower risk of over charge or discharge, wider temperature range of operation, longer lifespan, and being more environmentally friendly. ${ }^{(2-4)}$ In this study, a MFS is designed as a power buffer to facilitate the uniform generation of power by fuel cells. That is, the MFS provides additional power for starting, accelerating, and heavy loading, and absorbs the energy generated by braking.

The sections of this paper are: $\S 2$, design of hybrid powertrains, where the configurations of three types of hybrid powertrains are presented; $\S 3$, fuzzy controller design, in which the design of a fuzzy controller to flexibly regulate the charging/discharging power of MFS is discussed; $\S 4$, simulation studies based on Matlab/Simulink are described and an FTP-75 urban driving profile is carried out to confirm the applicability of the proposed powertrains and fuzzy control; and $\S 5$, in which several conclusions of this study are presented.

"Corresponding author: e-mail: kosono@mail.nutn.edu.tw 


\section{Design of Hybrid Powertrains}

In this study, control by the MFS is used to prevent the fuel cell from operating in a lowefficiency region. In this way, a MFS can provide peak power to an EV when it is suddenly required and also absorb energy in braking. This control is based on the fuzzy-logic decision to regulate power flow by detecting the various demands of vehicle load to maintain a constant output of the fuel cells during operation, as shown in Fig. 1, where MFS is treated as a power buffer. Three types of operations are illustrated in Figs. 2(a)-2(c). First in Fig. 2(a), if the EV load is larger than the fuel cell output, the MFS discharges power. In this case, the governing equation is

$$
P_{\mathrm{FC}}+P_{\mathrm{MFS}}=P_{\mathrm{v}},
$$

where $P_{\mathrm{FC}}$ is the output of the fuel cell, $P_{\mathrm{MFS}}$ is the charge/discharge power of the MFS, and $P_{\mathrm{v}}$ is the power demand of the vehicle.

On the other hand, in Fig. 2(b), if the EV load is smaller than the fuel cell output, the fuel cell maintains the same output, and excess power is stored by the MFS. Similarly in Fig. 2(c), when the EV in braking the regenerated power is stored by the MFS.

\subsection{Fuel-cell characteristics}

Using the electrochemical reaction of hydrogen and oxygen, a fuel cell generates electrical energy. First, the hydrogen is pressurized and provided to the anode. Next, when the hydrogen arrives at the anode catalytic layer, it splits into protons and electrons, which flow into an electrolytic membrane where the electrons are blocked and shunted to the cathode. Finally, through an external circuit, the electrons travel back to the cathode. Electric current is generated by this process. The electrochemical reaction in a fuel cell can be expressed as

$$
\mathrm{H}_{2}+\frac{1}{2} \mathrm{O}_{2} \rightarrow \mathrm{H}_{2} \mathrm{O}+\text { heat }+ \text { electricity. }
$$

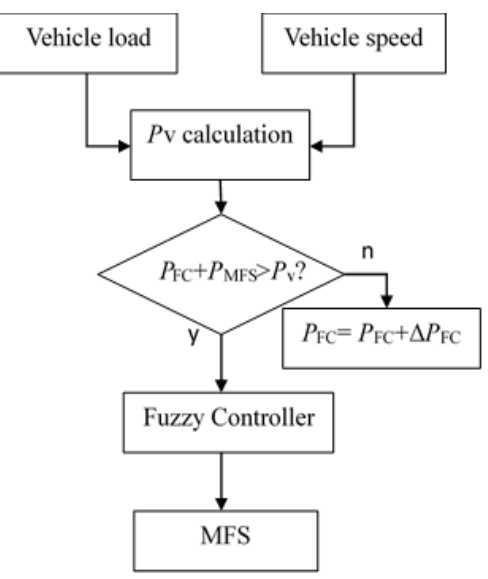

Fig. 1. Illustration of powertrain.

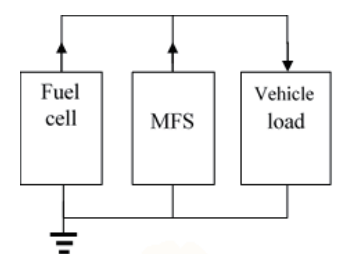

(a)

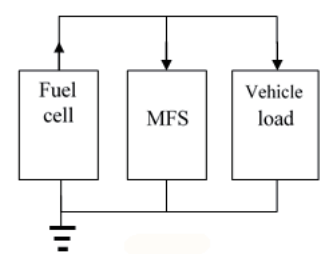

(b)

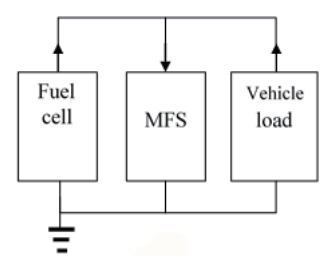

(c)

Fig. 2. Types of hybrid powertrains. 
In this study, the thermodynamic models of electrochemical and chemical reactions (i.e., chemical species dynamics, temperature) inside the fuel-cell stack are considered based on the following principles:

(1) balanced equations for the electrochemical model, ${ }^{(5)}$

(2) energy balance in the thermodynamic model, ${ }^{(6)}$

(3) Nernst equation relating to voltage activation, concentration, and ohmic losses. ${ }^{(7)}$

In addition, voltage losses resulting from ohmic losses, activation losses, and concentration losses are considered. In this study, the fuel cell is treated as a dc power source connected to a motor through de/ac inverters. In addition, conditions such as auxiliary system dynamics, electrochemical reactions inside the stack, fluid dynamics, gas diffusion, stack humidity, and temperature effects are also considered in the analysis of the power generation and the hydrogen consumption. ${ }^{(8)}$ To fit the polarization curve, the control strategy for fuel cell using the physical models listed is set as a 1.2-kW Nexa power module. To test the design and control process, a $48-\mathrm{V} \mathrm{dc}$ bus is chosen. The models and interface blocks of the fuel cell are simulated in Matlab/ Simulink.

\subsection{MFS characteristics}

The MFS architecture is shown in Fig. 3, featuring a likely-tunable-inertia cylindrical mass that allows kinetic energy to accumulate and be released. Magnetic bearings enable it to turn at high rotation speeds for reduced losses. The motor/generator coupled with a static converter is used for electromechanical energy conversion. These elements are placed in a safe vacuum sealed vessel to minimize friction losses. ${ }^{(2)}$

The MFS can be modeled as additional inertia connected to the shaft of the vehicle. The control target is to maintain flywheel operation at a constant value when the machine is magnetized. ${ }^{(9)}$ The swing equation governing the changes of the rotor speed can be expressed as

$$
J \frac{d \omega_{\mathrm{FMS}}}{d t}=T_{\mathrm{e}}
$$

where $J$ is the flywheel inertia, $\omega_{\mathrm{FMS}}$ is the rotational speed of the rotor, and $T_{\mathrm{e}}$ is the electrical torque of the machine, defined as

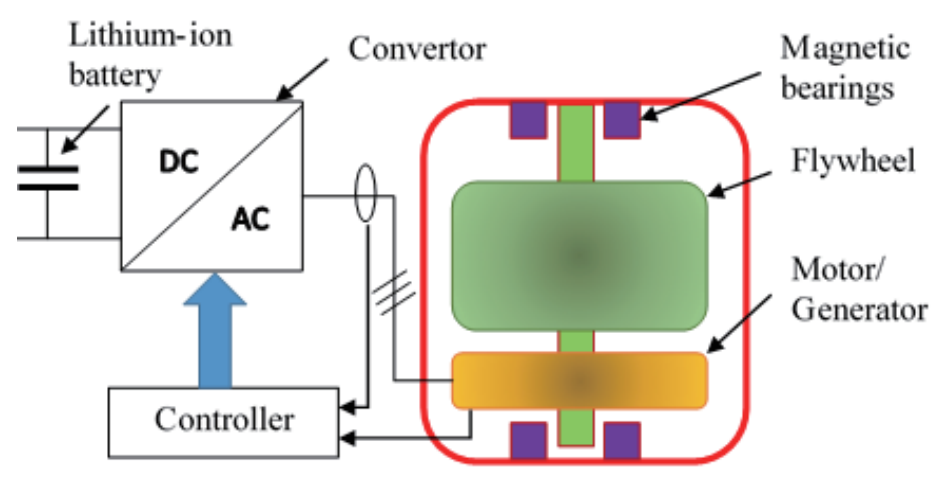

Fig. 3. (Color online) MFS structure. 


$$
T_{\mathrm{e}}=1.5 p(1-\sigma) L_{\mathrm{s}} i_{\mathrm{m}} i_{q}
$$

where $p$ is the pole-pair number, $\sigma$ is the total leakage coefficient, $L_{\mathrm{s}}$ is stator inductance, $i_{\mathrm{m}}$ is the magnetizing current, and $i_{\mathrm{q}}$ is the equilibrium value for current in field coordinates.

\section{Fuzzy Controller Design}

The controller is designed as a 2-input-1-output system. It has been successfully applied to many commercial products and industrial systems, most notably in automated control, management science, and artificial intelligence. Reference 10 discusses a tracking problem in which a tricycle mobile robot with two degrees of freedom and front wheel steering was solved using a fuzzy control. Reference 11 proposed a fuzzy-control approach to solve the nonlinear problem of electromagnetic suspension systems. In this study, fuzzy control is employed as the controller for the MFS system. The control variables of the system are defined as

$$
\begin{gathered}
\Delta P_{\mathrm{MFS}}(k)=P_{\mathrm{E}}(k)-P_{\mathrm{v}}(k), \\
\frac{d \Delta P_{\mathrm{MFS}}(k)}{d t}=P_{\mathrm{MFS}}(k)-P_{\mathrm{MFS}}(k-1),
\end{gathered}
$$

where $P_{\mathrm{MFS}}(k)$ and $d \Delta P_{\mathrm{MFS}}(k) / d t$ are the power difference and variation of MFS, respectively.

In Fig. 4, the membership function that is used to convert the input and output variables into linguistic variables for fuzzy inference is shown. The scaling factors Si with the subscript $i=1,2$, 3 represent the output error, the change in error of the system, and the control output, respectively. ${ }^{(10)}$ Table 1 shows the control rules for fuzzy decisions.

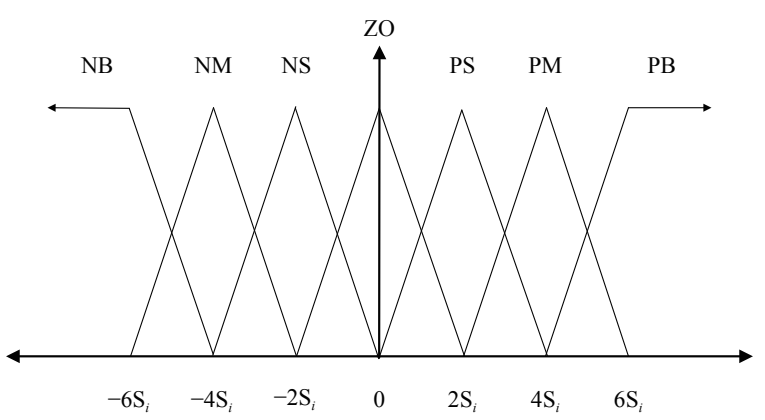

Fig. 4. Membership function of the fuzzy controller.

Table 1

Fuzzy rule table.

\begin{tabular}{lccccccc}
\hline \multirow{2}{*}{$\Delta$ shift $(k)$} & \multicolumn{7}{c}{ shift $(k)$} \\
\cline { 2 - 7 } & NB & NM & NS & ZO & PS & PM & PB \\
\hline NB & PM & PS & PS & ZO & PS & PM & PB \\
NM & PS & PS & PS & ZO & PS & PM & PM \\
NS & PS & PS & PS & ZO & PS & PS & PS \\
ZO & ZO & ZO & ZO & ZO & ZO & ZO & ZO \\
PS & NS & NS & NS & ZO & NS & NS & NS \\
PM & NM & NM & NS & ZO & NS & NS & NS \\
PB & NB & NM & NS & ZO & NS & NS & NM \\
\hline
\end{tabular}




\section{Simulation Studies and Confirmation}

For the effectiveness of the proposed control to be confirmed, a simulation using the FTP-75 urban driving cycle was carried out. In this study, the power consumed by the EV can be calculated by

$$
P_{\mathrm{v}}=\left(M \times g \times f_{\mathrm{r}}+\frac{1}{2} \rho_{\mathrm{a}} C_{\mathrm{D}} A_{\mathrm{f}} V^{2}+M \cdot \frac{d V}{d t}\right) \times V
$$

where $P_{\mathrm{v}}$ is the power consumption of the EV in watts, $M$ is the total mass of the EV in kilograms, $g$ is acceleration due to gravity $\left(9.81 \mathrm{~m} / \mathrm{s}^{2}\right), f_{\mathrm{r}}$ is the rolling resistance coefficient of the tires, $\rho_{\mathrm{a}}$ is the density of air $\left(1.202 \mathrm{~kg} / \mathrm{m}^{3}\right), C_{\mathrm{D}}$ is the coefficient of aerodynamic drag on the $\mathrm{EV}, A_{\mathrm{f}}$ is the front sectional area of the EV, and $V$ is the EV driving speed in meters per second. Table 2 shows the specifications of a sample vehicle in Ref. 12 which was used in the simulation.

As mentioned, this study uses the FTP75 urban drive cycle to confirm the effectiveness of MFS control. Using Eq. (8), the power consumption of the EV subjected to the FTP75 driving profile can be calculated. The power-consuming profile of $P_{\mathrm{v}}$ during driving is shown in Fig. 5. Based on the governing Eq. (1) and via the MFS control, the outputs of the MFS and fuel cell can be determined and are shown in Figs. 6 and 7, respectively. Note that, as the EV requires more power, the MFS supplies kinetic energy to maintain fuel-cell output at a close-to-constant value. As the power consumed by the EV is small, the MFS absorbs the kinetic energy to keep fuel cell output at a close-to-constant value. When the EV brakes, the MFS not only absorbs the kinetic energy from the EV but also charges the output power directly from fuel cell to the battery.

Table 2

Specification of the vehicle example.

\begin{tabular}{lc}
\hline EV mass $(\mathrm{kg})$ & 1450 \\
Resistance coefficient in rolling & 0.013 \\
Aerodynamic drag coefficient & 0.29 \\
Front sectional area $\left(\mathrm{m}^{2}\right)$ & 2.13 \\
Wheel radius $(\mathrm{m})$ & 0.28 \\
\hline
\end{tabular}

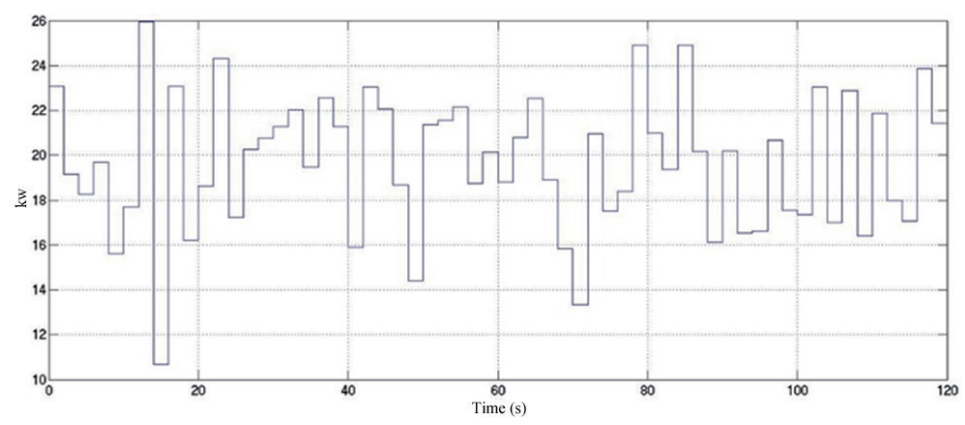

Fig. 5. EV loading based on the FTP-75 profile. 


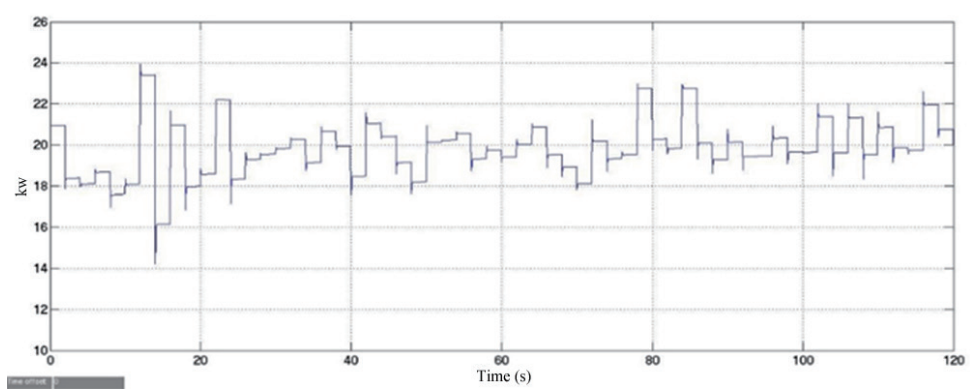

Fig. 6. Flywheel output.

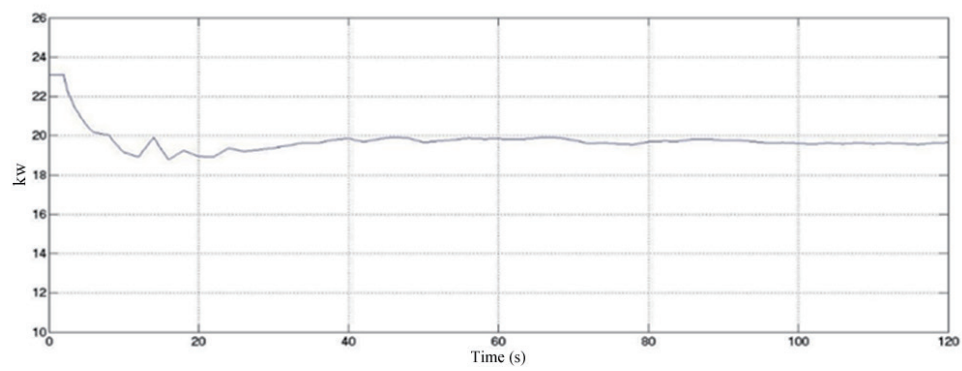

Fig. 7. Fuel cell output.

\section{Conclusions}

This paper describes a hybrid powertrain and MFS design based on fuzzy control to improve the disadvantages of fuel cells used in EVs. The effectiveness of the design is illustrated using a sample EV subjected to the FTP-75 urban drive cycle. Results of the simulation verify that the designed MFS can provide all loading required by the EV and maintain the power output of fuel cells at a close-to-constant value.

\section{Acknowledgements}

This work was supported by the National University of Tainan under Grant AB 104-124.

\section{References}

B. G. Pollet and J. L. Shang: Electrochim. Acta 84 (2012) 235.

2 G. N. Prodromidis and F. A. Coutelieris: Renewable Energy 39 (2012) 149.

3 C. Toh and S. Chen: Proc. IEEE Int. Conf. Magnetics (IEEE, Beijing, China, 2015) p. 1.

4 S. N. Motapon, L.-A. Dessaint, and K. Al-Haddad: IEEE Trans. Ind. Electron. 61 (2014) 1320.

5 L. Barelli, G. Bidini, and A. Ottaviano: Appl. Energy 110 (2013) 173.

$6 \quad$ K. J. Albrecht and R. J. Braun: J. Power Sources 304 (2016) 402.

7 W. Wua and D.-W. Jhaob: J. Process Control 22 (2012) 653.

8 C. Raga, A. Barrado, and A. Lazaro: IEEE Trans. Power Electron. 29 (2014) 5334.

9 R. L. Hockney and C. A. Driscol: Proc. IEEE Int. Conf. Telecommunications Energy (IEEE, Melbourne, Vic., 1997) pp. 105-109.

10 R. E. Precup, R. C. David, and E. M. Petriu: IEEE Trans. Ind. Inf. 8 (2012) 791.

11 X. Su, X. Yang, P. Shi, and L. Wu: Mechatronics 24 (2014) 328.

12 G. Park, S. Lee, S. Jin, and S. Kwak: Expert Syst. Appl. 41 (2014) 2595. 